\title{
COMPARAÇÃO DE TÉCNICAS DE CONCENTRAÇÃO POR SEDIMENTAÇÃO E COLORAÇÃO PARA DETECÇÃO DE OOCISTOS DE Cryptosporidium spp. EM AMOSTRAS FECAIS DE CÃES E GATOS
}

MOREIRA, Andrios da Silva '; BAPTISTA, Cristiane Telles "; BRASIL, Carolina Litchina ${ }^{1}$; VALENTE, Júlia de Souza ${ }^{1}$; BRUHN, Fábio Raphael Pascoti ${ }^{2}$; PEREIRA, Daniela Isabel Brayer ${ }^{2}$.

${ }^{1}$ Doutorando(a) do Programa de Pós-Graduação em Parasitologia, Instituto de Biologia, Universidade Federal de Pelotas; ${ }^{2}$ Professor(a), Faculdade de Veterinária, Universidade Federal de Pelotas.

\section{RESUMO}

$\mathrm{N}$ este estudo realizou-se a comparação e análise de concordância entre técnicas de concentração por sedimentação usando acetato de etila e formalina-éter, bem como se avaliou os métodos de Kinyoun, Ziehl-Neelsen e Safranina para detecção de oocistos de Cryptosporidium spp. em fezes de animais de companhia. A técnica da Auramina-O fenicada foi empregada como método de triagem. De 136 amostras de fezes analisadas, 32 (23,53\%) apresentaram oocistos de Cryptosporidium spp., sendo 29 (21,32\%) pela técnica de acetato de etila e $20(14,71 \%)$ pela técnica de formalina-éter. O teste Kappa evidenciou nível de concordância bom entre os métodos de concentração para a técnica da Auramina e concordância excelente para os métodos de Kinyoun, Ziehl-Neelsen e Safranina. Quando comparadas as técnicas, observou-se que houve fraca concordância entre os testes Kinyoun e Ziehl-Neelsen, e não houve concordância entre os métodos de Kinyoun ou ZiehlNeelsen quando comparados com a técnica de Safranina. Na impossibilidade de utilizar o método da Auramina-O fenicada, que necessita microscopia fluorescente, os resultados encontrados sugerem a utilização da técnica de concentração por sedimentação com acetato de etila seguida da técnica de Kinyoun para a identificação de oocistos de Cryptosporidium spp. O emprego de acetato de etila ou formalina-éter não influenciou os resultados para os métodos de coloração que utilizam microscopia de luz, embora oocistos mais íntegros tenham sido observados quando utilizado acetato de etila.

Palavras-chave: Criptosporidiose. Kinyoun. Ziehl-Neelsen. Safranina. 
Cryptosporidium spp. é um parasito epicelular oportunista que infecta o epitélio gastrointestinal de diversos hospedeiros, incluindo mamíferos, répteis, peixes e aves (THOMPSON et al., 2016).

Em países desenvolvidos, a atenção é maior para o diagnóstico e tratamento, além da prevenção da infecção por via hídrica e alimentícia. Já nos países em desenvolvimento, os esforços são direcionados para reduzir casos de doença em populações de risco, tais como: contato com animais de criação (principalmente bovinos), áreas de saneamento precário e sistema imune deficiente (THOMPSON et al., 2016).

Vários testes diagnósticos de coloração e microscopia são utilizados para detecção de oocistos de Cryptosporidium em amostras fecais de animais ou humanos. No entanto, não há uma metodologia padrão que tenha ampla aceitação pelos pesquisadores (CARVALHO, 2009).

A técnica de sedimentação por formalina-éter é frequentemente empregada para concentrar ovos, cistos e oocistos dos mais diversos parasitos intestinais, porém, o uso de éter tem a desvantagem de diminuir o número de oocistos visualizados na microscopia, bem como ocasionar distorções ou alterações em sua morfologia. Com o intuito de reduzir essas desvantagens, Young et al. (1979) propuseram a substituição do éter por acetato de etila e obtiveram melhores resultados da técnica.

O objetivo do presente estudo foi verificar a concordância entre as técnicas de coloração empregadas para deteç̧ão de oocistos de Cryptosporidium spp. em fezes de animais de companhia. Adicionalmente, comparar a eficiência entre as técnicas de concentração por sedimentação como método auxiliar para microscopia de fluorescência e de luz.

Amostras de fezes de animais de companhia, 116 cães e 20 gatos, foram coletadas em municípios da região sul do Rio Grande do Sul, totalizando 136 amostras. As amostras foram coletadas individualmente pelos respectivos tutores, logo após a evacuação, em seguida foram armazenadas em recipientes estéreis devidamente identificados. As amostras foram armazenadas e mantidas sob refrigeração até o seu processamento. 
No laboratório, todas as amostras de fezes analisadas foram previamente submetidas à retirada de sujidades. Aproximadamente $2 \mathrm{~g}$ de cada amostra foram diluídos em $10 \mathrm{~mL}$ de água destilada e submetidos à filtração em gaze e centrifugação por 3 minutos a 3000 rpm, sendo repetido o processo até a limpeza total do sobrenadante. Posteriormente, as fezes foram separadas em dois tubos tipo Falcon e processadas, empregando dois diferentes protocolos de sedimentação:

1) sedimento diluído em $2 \mathrm{~mL}$ de formalina $10 \%$ e adicionado de $3 \mathrm{~mL}$ de éter etílico, centrifugado a 3000 rpm por 3 minutos e o sobrenadante descartado;

2) sedimento diluído em $2 \mathrm{~mL}$ de formalina $10 \%$ e adicionado de $3 \mathrm{~mL}$ de acetato de etila, centrifugado a 3000 rpm por 10 minutos e o sobrenadante desprezado.

Para triagem das amostras de fezes, empregou-se o método da Auramina-O fenicada. As amostras que, nesse método, apresentaram oocistos de Cryptosporidium spp. foram, posteriormente, analisadas pelos métodos de coloração por microscopia de luz, incluindo o método de Kinyoun (Ky), Ziehl-Neelsen (ZN) modificado e Safranina (S).

Uma alíquota de $10 \mu \mathrm{L}$ do sedimento de cada protocolo de sedimentação foi utilizada para a confecção dos esfregaços e coloração por Auramina-O fenicada. Os esfregaços foram fixados no calor, corados com auramina-O e submetidos à secagem à temperatura ambiente durante 15 minutos. Logo após, foram lavados com água destilada e, posteriormente, descorados com álcool-ácido durante 2 minutos, seguido por novo processo de lavagem. No passo seguinte, os esfregaços foram cobertos com permanganato de potássio durante 2 minutos e uma nova etapa de secagem à temperatura ambiente foi realizada, para então serem examinados ao microscópio de fluorescência em objetivas de 10X e 40X.

No método de Kinyoun, após a realização dos esfregaços, as lâminas foram submetidas à secagem à temperatura ambiente, fixadas com metanol por 5 minutos e submetidas à secagem a $37{ }^{\circ} \mathrm{C}$ durante 5 minutos. Posteriormente, as lâminas foram coradas com fucsina básica (corante de Kinyoun) durante 20-30 minutos, seguida de descoloração por ácido sulfúrico $2 \%$ por 30-40 segundos e lavagem em água corrente. Na sequência, as lâminas foram contra-coradas com verde malaquita 5\% durante 1 minuto, lavadas em água corrente 
e secas em temperatura ambiente. A leitura foi realizada em microscopia de luz em objetiva de imersão (100X).

Para a realização do método de Ziehl-Neelsen modificado, as lâminas foram fixadas ao ar e coradas com carbol-fucsina durante 15 minutos. Logo após, foram lavadas com água corrente, descoradas em metanol ácido $1 \%$ por 10-15 segundos e novamente lavadas em água corrente. Posteriormente, foram contra-coradas com verde malaquita $0,4 \%$ durante 30 segundos, submetidas à lavagem em água corrente e secagem à temperatura ambiente. Logo após, foram examinadas em microscopia de luz em objetiva de imersão (100X).

No método de Safranina, as lâminas foram fixadas com metanol clorídrico a $1 \%$ durante 3-5 minutos. Após secagem à temperatura ambiente, as lâminas foram coradas com safranina aquosa $1 \%$ durante 1 minuto e aquecidas em chama do bico de Bunsen até fervura da solução. Após lavagem em água destilada, as lâminas foram contra-coradas com azul de metileno 1\% durante 30 segundos, lavadas com água destilada e secas em temperatura ambiente para a avaliação em microscopia de luz com objetiva de imersão (100X).

Para análise estatística indutiva, foi utilizado o software SPSS 20.0. O coeficiente Kappa de Cohen (K) e o intervalo de confiança a 95\% (IC. 95\%) foram utilizados como indicadores de concordância ajustados entre os resultados obtidos pelos diferentes testes para diagnóstico de Cryptosporidium spp. em amostras de fezes de cães e gatos. De acordo com o valor pontual obtido pelo $K$, as relações foram classificadas como excelente $(>0,75)$, boa $(0,75-$ $0,40)$ ou fraca $(<0,40)$ concordância (PESTANA; GAGEIRO, 2008). Considerou-se um nível mínimo de confiança de $95 \%$ em todas as análises estatísticas realizadas.

Oocistos corados por Auramina-O fenicada apresentaram-se de forma esférica, aproximadamente $5 \mu \mathrm{m}$ de diâmetro, fluorescentes, destacando-se em fundo escuro. Não foi possível visualizar as estruturas internas em objetiva de 40X. No método de Kinyoun, os oocistos apresentaram-se esféricos, $5 \mu \mathrm{m}$ de diâmetro, coloração rosa claro com estruturas internas escuras visualizadas pela objetiva de 100X. Na técnica de Ziehl-Neelsen, coraram-se em vermelho escuro a roxo, $5 \mu \mathrm{m}$ de diâmetro, com estruturas internas coradas em tom mais escuro, sendo observados em objetiva de 100X. Características similares foram observadas pela coloração de Safranina, porém os oocistos coraram-se em vermelho claro. 
A deteç̧ão de oocistos de Cryptosporidium spp. em amostras de fezes, água e outros materiais é difícil, dependente de padronização de técnicas e da experiência dos profissionais (CARVALHO, 2009). Técnicas imunológicas e moleculares têm sido desenvolvidas para diagnóstico, apresentando resultados mais precisos, porém o alto custo limita sua utilização (ROSSLE; LATIF, 2013). Considerando o tamanho reduzido dos oocistos, somado a métodos trabalhosos e caros, estudos têm sido desenvolvidos com o objetivo de melhorar as técnicas para detecção do parasito, todavia, apresentam resultados variáveis (INÁCIO, 2012). No presente estudo, empregou-se a técnica da Auramina-O fenicada como teste de triagem para avaliar a presença de oocistos de Cryptosporidium spp. em fezes de cães e gatos, uma vez que essa técnica é considerada metodologia padrão no Reino Unido (HANSCHEID; VALADAS, 2008). Através desse método, observou-se que, do total de 136 amostras de fezes analisadas, 32 (23,53\%) apresentaram oocistos de Cryptosporidium spp., sendo 29 (21,32\%) identificados em lâminas previamente confeccionadas com acetato de etila e $20(14,71 \%)$ em lâminas previamente confeccionadas com formalina-éter (Tabela 1), apresentando boa concordância entre as técnicas de concentração por sedimentação pelo teste Kappa (Tabela 2).

Tabela 1 - Resultados descritivos dos métodos de coloração.

\begin{tabular}{lccc} 
Técnica de coloração & $(+) \mathrm{FE}$ & $(+) \mathrm{AE}$ & Total $(+)$ \\
\hline $\begin{array}{l}\text { Auramina-O fenicada* } \\
\text { Kinyoun }\end{array}$ & 9 & 29 & 32 \\
& & 10 & 11 \\
Ziehl-Neelsen modificado & 7 & 7 & 8 \\
& & & \\
Safranina & 5 & 7 & 8 \\
\hline FE: formalina-éter; AE: acetato de etila. & & \\
*Padrão ouro (triagem). &
\end{tabular}

Os resultados obtidos no teste Kappa, demonstraram, também, que o uso de ambos os métodos de concentração por sedimentação não apresentou diferenças quando as técnicas 
de coloração foram analisadas isoladamente, posto que a concordância foi excelente. Esses resultados discordam dos relatados por Casemore et al. (1985) que encontraram maior sensibilidade utilizando formalina-éter em testes de Auramina e Ziehl-Neelsen para detecção de Cryptosporidium spp. e outros patógenos em material fecal humano. Diferiram também de Young et al. (1979) que propuseram o acetato de etila como fator favorável na detecção de oocistos, cistos e ovos de parasitos em microscopia.

Tabela 2 - Concordância entre os métodos de concentração por sedimentação e coloração para detecção de oocistos de Cryptosporidium spp. em fezes de animais de companhia ( $n=136)$.

\begin{tabular}{|c|c|c|c|}
\hline Comparação entre métodos & $P$ & $\mathrm{~K}^{* *}$ & IC.95\% \\
\hline Ky-ae x Ky-fe* & 0,000 & 0,833 & $0,605-1,0$ \\
\hline Ky-ae x ZN-ae* & 0,026 & 0,391 & $0,013-0,769$ \\
\hline Ky-ae x ZN-fe* & 0,026 & 0,391 & $0,013-0,769$ \\
\hline Ky-ae x S-ae & 0,117 & - & - \\
\hline Ky-ae x S-fe & 0,601 & - & - \\
\hline Ky-fe x ZN-ae* & 0,026 & 0,391 & $0,013-0,769$ \\
\hline Ky-fe x ZN-fe* & 0,026 & 0,391 & $0,013-0,769$ \\
\hline Ky-fe x S-ae & 0,601 & - & - \\
\hline K-fe x S-fe & 0,601 & - & - \\
\hline ZN-ae x ZN-fe* & 0,000 & 0,817 & $0,569-1,0$ \\
\hline ZN-ae x S-ae & 0,451 & - & - \\
\hline ZN-ae x S-fe & 0,064 & - & - \\
\hline ZN-fe x S-ae & 0,451 & - & - \\
\hline ZN-fe $\times$ S-fe & 0,064 & - & - \\
\hline S-ae X S-fe* & 0,000 & 0,795 & $0,517-1,0$ \\
\hline
\end{tabular}

\footnotetext{
*significância estatística $(p<0,05) ;$ Ky = Kinyoun; ZN = Ziehl-Neelsen; S = Safranina; ae = acetato de etila; fe = formalina-éter.

**Valores de K (Kappa): >0,75 - concordância excelente; 0,75-0,40 - concordância razoável a boa; <0,4 concordância fraca (PESTANA; GAGEIRO, 2008).
} 
Quando comparadas as técnicas, observou-se que: houve fraca concordância entre os testes Kinyoun e Ziehl-Neelsen, e não houve concordância entre os métodos de Kinyoun ou ZiehlNeelsen quando comparados com a técnica de Safranina, independente do uso de formalina-éter ou acetato de etila como solvente no método de concentração (Tabela 2). Ao comparar a concordância pelo teste de Kappa entre Kinyoun e um método imunoenzimático, McCluskey et al. (1995) encontraram concordância intermediária entre os dois métodos $(K=0,42)$, sendo o método imunoenzimático mais sensível do que o Kinyoun, entretanto, na avaliação de poucas amostras este último é mais econômico. Rodrigues et al. (2016) ao utilizarem os métodos de Ziehl-Neelsen e Safranina, na pesquisa de oocistos de Cryptosporidium spp. em amostras fecais de 152 bezerros, encontraram maior positividade pela técnica de ZN.

Na impossibilidade de utilizar o método da Auramina-O fenicada, que necessita microscopia fluorescente, para verificar a presença de oocistos de Cryptosporidium spp, os resultados encontrados, neste experimento, sugerem a utilização da técnica de concentração por sedimentação com acetato de etila seguida da técnica de Kinyoun. No entanto, estudos mais detalhados devem ser realizados com o objetivo de se definir qual técnica de coloração para microscopia de luz deve ser utilizada como rotina laboratorial para deteç̧ão de oocistos de Cryptosporidium spp.

Em relação as duas técnicas de concentração por sedimentação, avaliadas através do teste Kappa, os resultados obtidos demonstraram que o emprego de acetato de etila ou formalina éter para a concentração de oocistos por sedimentação não alterou significativamente os resultados. No entanto, cabe ressaltar que os autores verificaram que em lâminas confeccionadas com acetato de etila como solvente foi possível identificar-se oocistos mais íntegros e em maior número do que em lâminas confeccionadas utilizando formalina-éter. 


\section{COMPARISON OF CONCENTRATION BY SEDIMENTATION AND STAINING TECHNIQUES FOR THE DETECTION OF OOCYSTS OF Cryptosporidium spp. IN FECAL SAMPLES FROM DOGS AND CATS}

\section{ABSTRACT}

T he present study evaluated a comparison and concordance analysis between concentration techniques using ethyl acetate and formalin-ether, as well as comparing Kynioun, Ziehl-Neelsen and Safranin methods to detect Cryptosporidium spp. oocysts in feces from dogs and cats. The Auramine-phenol method was used as a screening method. From the fecal samples analyzed, 32 (23.53\%) presented Cryptosporidium spp. oocysts, 29 (21.32\%) by the ethyl acetate technique and $20(14.71 \%)$ by the formalin-ether technique. The Kappa test showed good agreement between the concentration methods for the Auramine technique and excellent agreement for the Kinyoun, Ziehl-Neelsen and Safranina methods. When comparing the techniques, it was observed that there was a poor agreement between the Kinyoun and Ziehl-Neelsen tests, and there was no agreement between the Kinyoun or Ziehl-Neelsen methods when compared with the Safranina technique. Since it is impossible to use the Auramine-phenol method, which requires fluorescent microscopy, the results suggest the use of the ethyl acetate sedimentation concentration technique followed by the Kinyoun technique to identify Cryptosporidium spp. The use of ethyl acetate or formalin ether did not influence the results for staining methods that use light microscopy, although more intact oocysts were observed when using ethyl acetate.

Keywords: Cryptosporidiosis. Kinyoun. Ziehl-Neelsen. Safranin.

\section{COMPARACIÓN DE TÉCNICAS DE CONCENTRACIÓN POR SEDIMENTACIÓN Y COLORACIÓN PARA DETECCIÓN DE OOQUISTES DE Cryptosporidium spp. EN MUESTRAS FECALES DE PERROS Y GATOS}

\section{RESUMEN}

\footnotetext{
E n este estudio se realizó la comparación y el análisis de concordancia entre las técnicas de concentración por sedimentación con acetato de etilo y formol-éter, y se evaluaron los métodos de Kinyoun, Ziehl-Neelsen y Safranina para la detección de ooquistes de
} 
Cryptosporidium spp. en heces de animales de compañía. La técnica de Auramina se utilizó como método de clasificación. De 136 muestras de heces analizadas, 32 (23,53\%) presentaron ooquistes de Cryptosporidium spp., 29 (21,32\%) por la técnica de acetato de etilo y $20(14,71 \%)$ por la técnica de formol-éter. La prueba Kappa evidenció un buen nivel de concordancia entre los métodos de concentración por la técnica de Auramina y una concordancia excelente para los métodos de Kinyoun, Ziehl-Neelsen y Safranina. Al comparar las técnicas, se observó que había una débil concordancia entre las pruebas Kinyoun y Ziehl-Neelsen, y no hubo concordancia entre los métodos Kinyoun o Ziehl-Neelsen en comparación con la técnica Safranina. En la imposibilidad de utilizar el método de Auramina, que requiere microscopía fluorescente, los resultados sugieren el uso de la técnica de concentración por sedimentación con acetato de etilo seguida de la técnica de Kinyoun para identificar Cryptosporidium spp. El uso de acetato de etilo o formol-éter no influyó en los resultados para los métodos de tinción que usan microscopía óptica, aunque se observaron oocistos más íntegros cuando fue utilizado acetato de etilo.

Palabras clave: Criptosporidiosis. Kinyoun. Ziehl-Neelsen. Safranina.

\section{REFERÊNCIAS}

CARVALHO, T. T. R. Estado atual do conhecimento de Cryptosporidium e Giardia. Revista de Patologia Tropical, v. 38, n. 1, p. 1-16, 2009.

CASEMORE, D. P.; ARMSTRONG, M.; SANDS, R. L. Laboratory Diagnosis of Cryptosporidiosis. Journal of Clinical Pathology, v. 38, n. 12, p. 1337-1341, 1985.

HANSCHEID, T.; VALADAS, E. Diagnosis of cryptosporidiosis using PCR or auramine $O$ with LED fluorescent microscopy: Which end of the stick? Acta Tropica, v. 109, n. 3, p. 247-248, 2009.

INÁCIO, S. V.; BRITO, R. L. L.; ZUCATTO, A. S.; et al. Cryptosporidium spp. infection in mares and foals of the northwest region of São Paulo State, Brazil. Revista Brasileira de Parasitologia Veterinária, v. 21, n. 4, p. 355-358, 2012.

MCCLUSKEY, B.; GREINER, E. C.; DONOVAN, G. A. Patterns of Cryptosporidium oocyst shedding in calves and a comparison of two diagnostic methods. Veterinary Parasitology, $v$. 60, n. 3-4, p. 185-190, 1995.

PESTANA, M. H.; GAGEIRO, J. N. Análise de dados para ciências sociais: a complementariedade do SPSS. 5. ed. Lisboa: Sílabo, 2008. 692p.

RODRIGUES, R. D.; GOMES, L. R.; SOUZA, R. R.; et al. Comparação da eficiência das colorações de Ziehl-Neelsen modificado e Safranina modificada na detecção de oocistos de 
Cryptosporidium spp. (Eucoccidiorida, Cryptosporidiidae) a partir de amostras fecais de bezerros de 0 a 3 meses. Ciência Animal Brasileira, v. 17, n. 1, p. 119-125, 2016.

ROSSLE, N. F.; LATIF, B. Cryptosporidiosis as threatening health problem: A review. Asian Pacific Journal of Tropical Biomedicine, v. 3, n. 11, p. 916-924, 2013.

THOMPSON, R. C. A.; KOH, W. H.; CLODE, P. L. Cryptosporidium - What is it? Food and Waterborne Parasitology, v. 4, p. 54-61, 2016.

YOUNG, K. H.; BULLOCK, S. L.; MELVIN, D. M.; et al. Ethyl Acetate as a Substitute for Diethyl Ether in the Formalin-Ether Sedimentation Technique. Journal of Clinical Microbiology, $v$. 10, n. 6, p. 852-853, 1979. 